\title{
Solar Spectral Fine Structure in 18-23 GHz Band
}

\author{
J. R. Cecatto ${ }^{1}$, K. R. Subramanian ${ }^{2 *}$, and H. S. Sawant ${ }^{1}$ \\ ${ }^{1}$ Instituto Nacional de Pesquisas Espaciais-INPE-CEP 12.201-970 - C.P. 515 , \\ São José dos Campos, SP, Brazil \\ ${ }^{2}$ Indian Institute of Astrophysics, Bangalore, India \\ Received 16 April, 1999
}

\begin{abstract}
On 30th June 1989 high sensitivity-spectral resolution observations of solar radio bursts were carried out in the frequency range of $18-23 \mathrm{GHz}$. The burst observed at 17:46 UT was different from the 60 bursts observed so far in the sense that it exhibited a frequency fine structure superimposed on the ongoing burst in its rising phase, i.e. an additional enhancement of the flux density of the order of 10 SFU, observed only in the 21 and $22 \mathrm{GHz}$ frequency channels, lasting for about $4 \mathrm{~s}$. Interaction of an emerging loop with an adjacent loop accelerated particles in that loop from which the broadband burst was emitted due to the gyrosynchrotron emission. The observed fine structure is interpreted as due to thermal gyro-emission at 6 th harmonic of the gyrofrequency originated from a hot kernel with short lifetime located at the top of emerging loop. We derived the hot kernel source parameters, the temperature as $8 \times 10^{7} \mathrm{~K}$, the magnetic field as $\simeq 1250 \mathrm{G}$ and the density as $\simeq 5 \times 10^{12} \mathrm{~cm}^{-3}$.
\end{abstract}

\section{Introduction}

High spectral-time resolution studies of solar active regions and of radio bursts at $m m$ wavelengths are scanty. Kaverin et al. (1979), for the first time investigated the spectra of active regions with $60 \mathrm{MHz}$ frequency resolution in the frequency range of 5-7 GHz and reported frequency fine structures with a bandwidth of 150-400 $\mathrm{MHz}$. This group extended the frequency range of observations to $8-12 \mathrm{GHz}$ and reported the existence of frequency fine structure in the spectra of the active region in this band also (Kaverin et al. 1980). Later on, Lang and Willson (1983) reported fine structure emission in active regions in the frequency range of $1375-$ $1720 \mathrm{MHz}$. Schmahl et al. (1984) reported steep spectra in active regions in the band of 4496 to $4966 \mathrm{MHz}$. Observations of active regions using VLA at 10 closely spaced frequencies between 1440 and $1720 \mathrm{MHz}$, with a spatial resolution of $\sim 4$ arc seconds by Willson (1985) showed significant changes in the brightness temperature over a narrow frequency range. He attributed them to cyclotron line emission from a narrow layer where the temperature is elevated. Recently, Bogod et al., (1998) using the $\mathrm{RATAN}-600$ radio telescope in the frequency range of $937.5 \mathrm{MHz}-17.6 \mathrm{GHz}$ also observed narrowband emission, similar to Willson's observations, but around $3330 \mathrm{MHz}$ with bandwidth of $\sim 750 \mathrm{MHz}$.

Stähli et al. (1989, 1990), using the Owen's Valley Frequency Agile Interferometer operating between 1$18 \mathrm{GHz}$ with 10 seconds time resolution, showed the existence of frequency fine structures in the spectra of the solar bursts. For the first time, Gontarev and Klassen (1987) and Klassen (1992) reported frequency fine structures in the rising part of the microwave burst associated with the impulsive phase of a flare, with fractional bandwidth of $\Delta f / f \leq 0.1$ and intensity $\Delta S / S \simeq 10 \%$, in the frequency range of $2100-3100$ MHz. Fridman et al. (1994) reported "blip-like" emission in the initial phase of the bursts in the frequency range of $8-12 \mathrm{GHz}$ with a bandwidth of about $2-4$ $\mathrm{GHz}$ and positive drift rate of $2 \mathrm{GHz} / \mathrm{s}$. They suggested that these emissions are due to plasma mechanism. For the first time, we report evidence for the existence of frequency fine structure still at higher frequencies than reported earlier and discuss possible model for its origin.

\section{Observations}

Microwave observations of solar active regions and radio bursts are being carried out since 1988 by using the variable frequency $(18-23 \mathrm{GHz})$ radiometer and $13.7 \mathrm{~m}$

\footnotetext{
${ }^{*}$ On leave from Indian Institute of Astrophysics
} 
parabolic dish of Itapetinga Radio Observatory, Brazil. This system has a high sensitivity of 0.03-0.06 SFU, frequency resolution of $1 \mathrm{GHz}$, and time resolution of $0.6-$ 1.2 seconds. The spatial resolution is $\sim 4$ arc minutes at $21 \mathrm{GHz}$. Other details of the system and calibration procedures are given by Sawant and Cecatto (1994). For the observations reported here the time resolution was $1.2 \mathrm{~s}$.

In the three years of observations of active regions, with a total of about 500 hours, only two active regions showed evidence of frequency fine structures in their spectra with a bandwidth of about $2.0 \mathrm{GHz}$ lasting up to 90 minutes (Cecatto, 1990; Sawant et al. 1994). During the period of June-July, 1989, we have observed 30 microwave bursts, 11 of them on 30th June, 1989 when the antenna was tracking the active region $A R$ 5569 which had a magnetic field of 2100-2500 G at the photospheric level (Coffey; SGD 540-I, 1989) and was located at N19 W24. Out of the 11 bursts observed, only one showed frequency fine structure.

The burst reported here, exhibiting frequency fine structure, started at 17:46:01 UT and showed maximum intensity at 17:46:10 UT. Microwave (18-23 GHz) time profiles of this burst are shown in Fig. 1a. The differentiated intensity time profile plot which brings out the presence of frequency fine structure at 21 and $22 \mathrm{GHz}$ more clearly is shown in Fig. 1b. The burst duration is defined as the time interval when the signal is above $3 \sigma$ level, where $\sigma$ is rms noise. The total duration of the broad band burst is $\simeq 20$ seconds and its peak intensity is about 90 SFU. A frequency fine structure can be seen before the maximum at 17:46:06 UT, superposed on the broadband burst. This narrow band structure has a bandwidth of about $2 \mathrm{GHz}$, intensity of $10 \mathrm{SFU}$, and total duration of $\sim 4$ seconds.

This burst was associated with $\mathrm{H}-\alpha$ flare of SN type and soft X-ray M2.1 flare as observed by GOES satellite begining around 17:37 UT. The UltraViolet Spectrometer Polarimeter (UVSP) on board the Solar Maximum Mission satellite also observed this burst. It started around 17:45:30 UT, showed a peak after 17:46 UT and lasted for $\sim 2.5$ minutes (Schwartz, 1992).

Occurrence of fine structure in the microwave burst and/or its spectra reported up to $1-18 \mathrm{GHz}$ by various authors are mentioned in the introduction. Our observation suggests that they can be observed up to 22 GHz. Nine of the bursts observed by us on June 30th, 1989 were found to be associated with hard X-rays and 5 of them were found to be associated with Owen's Val- ley observations in the frequency range of (1-18) $\mathrm{GHz}$. Six of these bursts were analyzed with Hard X-ray data of Solar Maximum Mission satellite, and three showed one to one correlation in intensity and time with each time structure observed in the high energy ( $\geq 200 \mathrm{keV})$ bursts (Sawant et al., 1993). This leads us to believe that the observed fine structure is of real solar origin.
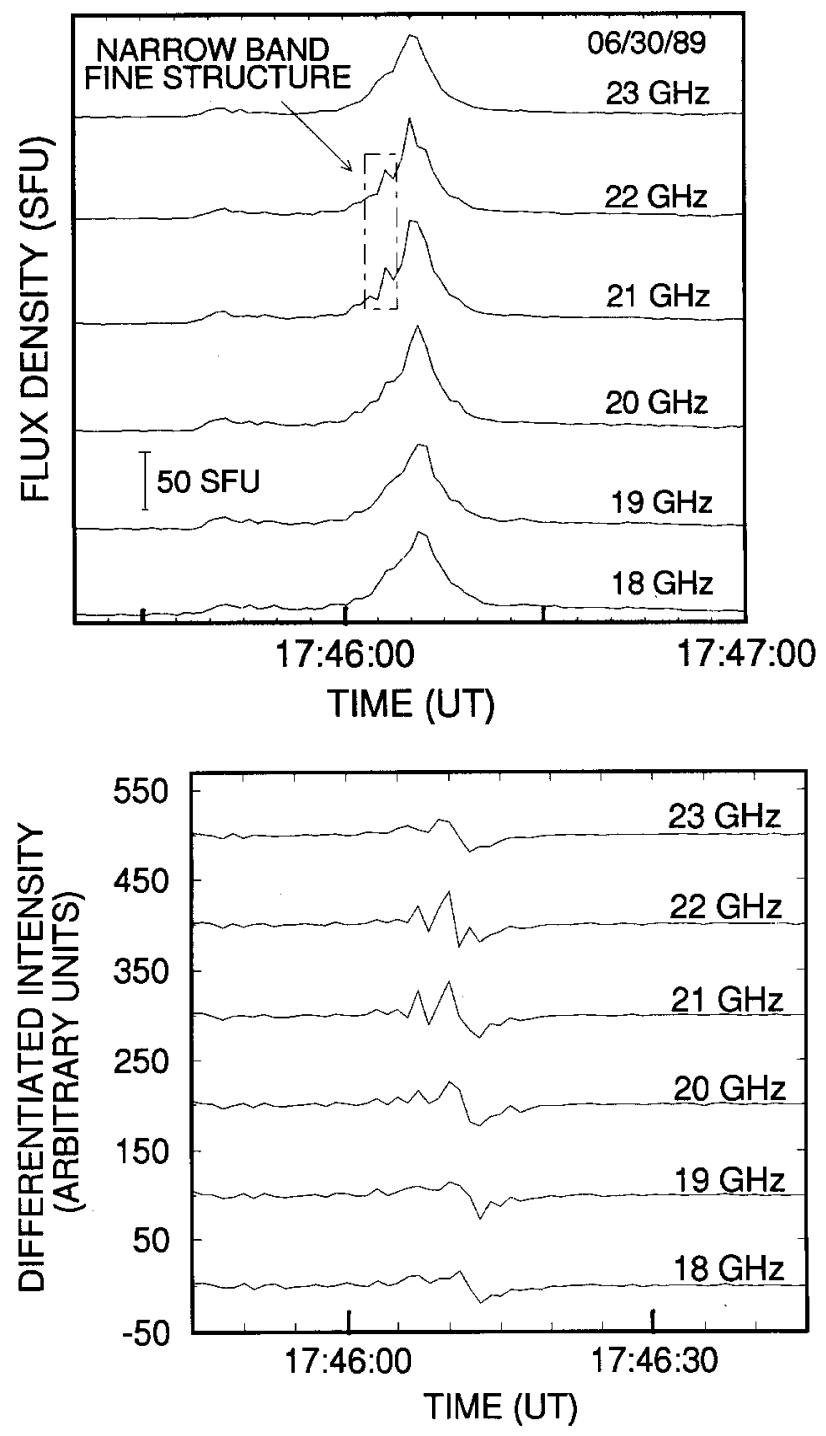

Figure 1. (TOP) Time profiles of a microwave (18-23 GHz) solar burst observed on June 30, 1989 at $\sim 17: 46$ UT with a frequency fine structure in $21-22 \mathrm{GHz}$ during the rise time. (BOTTOM) Differentiated time profiles at all six frequencies exhibiting more clearly the fine structure in 21 and 22 $\mathrm{GHz}$.

\section{Discussions and Interpreta- tion}

As mentioned in the introduction, fine structures in the active regions and bursts spectra in the microwave 
range have been reported since 1980 . At the same time, Zheleznyakov and Tikhomirov (1982) theoretically suggested that hot kernels located on the top of a magnetic loop with dimensions up to 6 arc seconds can emit thermal cyclotron radio emission and bremsstrahlung which may contain a set of lines at cyclotron harmonics and the maximum at plasma frequency. Lifetime of these emissions can be smaller than a couple of minutes or some seconds. These lines can appear as fine structure superimposed on the spectra of the active region and/or on the bursts and can be detected with an antenna having reasonably good sensitivity and spatial resolution of $\sim$ few arc minutes as is in our case, whereas the detection of cyclotron lines reported by Willson (1985) and Bogod et al. (1998) essentially requires high spatial resolution of the order of a couple arc seconds.

There is enough evidence that hot kernel or microwave sources lasting for a couple of seconds do exist. Recently, observations of transient bright points in Xrays have been reported by Kundu et al. (1994), and Van Driel-Gesztelyi (1996). Observations of transient brightening at X-ray wavelengths lasting for about 2 min. (Shimizu, 1995) and transient microwave brightening at $15 \mathrm{GHz}$ lasting for about $30 \mathrm{~s}$ (could be less due to VLA's poor time resolution) by Gopalswamy et al. (1994) support the possible existence of such short lived sources. Observations of transient microwave brightening lasting for seconds and their comparison with brightening in soft X-rays were noticed by Gopalswamy et al. (1994).

We suggest that the observed fine structure can originate from hot kernel's radio emission, lasting for a couple of seconds. However, we do not rule out the possibility that it may be due to plasma emission since our limited spectral-time resolutions do not permit us to investigate possible drift rates, as observed by Fridman et al. (1994) for the interpretation of their observations in the frequency range of 8-12 $\mathrm{GHz}$.

The proposed model for interpretation of the observed fine structure is shown in Fig. 2. The emerging loop (B) interacts with the adjacent loop (A) and accelerates the particles which generate the broadband $(18-23 \mathrm{GHz})$ radio emission in loop (A). The hot kernel lasting for $\leq 4 \mathrm{~s}$ in loop (B) generates a set of cyclotron line emission at 6 th harmonic which is the observed narrow band structure as superposed on the broadband emission since antenna's beamwidth is large enough to cover the whole active region including both the loops. Typical values of temperature and density for the remainder of loop (B) are $10^{7} \mathrm{~K}$ and $10^{11} \mathrm{~cm}^{-3}$ respectively. Values for the surroundings of the loop are $10^{6} \mathrm{~K}$ and $10^{9} \mathrm{~cm}^{-3}$.

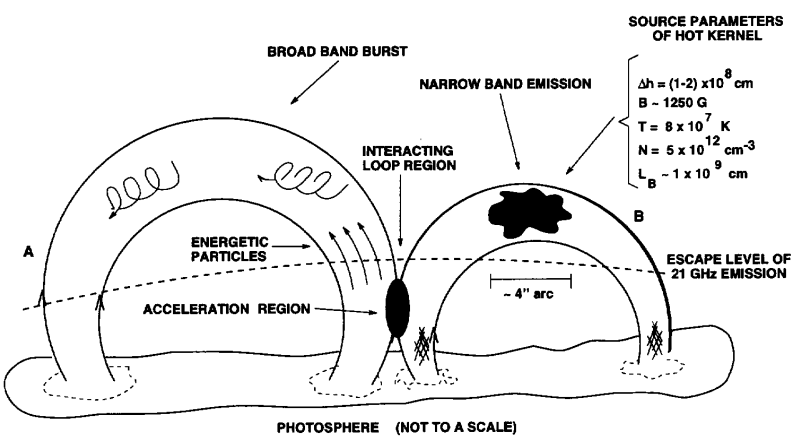

Figure 2. Simplified sketch for the magnetic configuration in the source. Broadband burst is produced in the loop (A) while the kernel located in the emerging loop (B) is responsible for the frequency-time fine structure.

It is well known that radio emission can escape above $2^{\text {nd }}$ harmonic and in case of hot kernel depending upon the kernel parameters it can emit higher harmonic. The estimated magnetic field values up to $5^{\text {th }}$ harmonic are $\geq 1500 \mathrm{G}$, however, at the height of escape level of $22 \mathrm{GHz}$ radio emission they are not consistent with the observed magnetic field of the associated active region. At the $6^{\text {th }}$ harmonic the order of magnetic field at escape level is $\simeq 1250 \mathrm{G}$, and is consistent with the observed photospheric magnetic field, 2300 $\mathrm{G}$, of the associated active region AR 5569. Therefore, we assume the lowest harmonic, $6^{t h}$, at which the fine structure emission can be originated.

According to Zheleznyakov and Tikhomirov (1982) the conditions of radiation escape at frequency $\omega=s \omega_{B}$ are given by:

$$
\frac{\omega_{p}^{2}}{\omega_{B}^{2} \sin ^{2} \alpha_{0}} \leq s(s \pm 1)
$$

where $\omega_{p}$ is the angular plasma frequency, $\omega_{B}$ is angular gyrofrequency, $s$ is harmonic number and $\alpha_{0}$ is the angle between the ray path and the magnetic field. For $N_{e} \simeq 5 \times 10^{12} \mathrm{~cm}^{-3}, B=1250 \mathrm{G}$ and $\alpha_{0}=1 \mathrm{rd}$, we can estimate that radiation escape occurs at $s \geq 7$ for the ordinary mode and $s \geq 6$ for the extraordinary. However, optical depth for ordinary mode is lower than that for the extraordinary as is discussed in detail ahead. Therefore, our assumption the observed radiation is at the 6 th harmonic is reasonable. We estimate the temperature, electron density, magnetic field and its variation through the kernel and its thickness from 
our observations. Assuming that the narrow band emission is generated at the 6 th harmonic in $21 \mathrm{GHz}$ for the extraordinary mode, the value of estimated magnetic field in the kernel is $\sim 1250 \mathrm{G}$.

Radio emission at $21 \mathrm{GHz}$ can escape from a height of $\geq 1 \times 10^{8} \mathrm{~cm}$ just above of the photosphere under assumptions that emission is at least at second harmonic and assumed density model is ten times that of Krinberg and Teplitskaja (1972). These authors provide a table with the physical parameters - electron density, temperature, height, etc - in the outer solar layers at maximum of solar activity.

The observed and suggested source sizes in the frequency range of 15 to $23 \mathrm{GHz}$ are of the order of $2-10$ arcsec (De Jager, 1979; Marsh \& Hurford, 1980; Gopalswamy et al. 1994). Smaller source sizes of the order of 2 arcsec will increase the brightness temperature to the order of about $5 \times 10^{8} \mathrm{~K}$, and will be the opposite in the case of bigger source sizes for the observed flux value of the fine structure. Hence, we assume a source size of the order of 4-5 arcsec. From the observed flux density of $10 \mathrm{SFU}$ for the fine structure and assuming source size of 4 arc seconds for the kernel, the estimated brightness temperature is $8 \times 10^{7} \mathrm{~K}$. Temperature of this order has been reported from X-ray kernels (Shimizu, 1995) and from microwave arcsec resolution observations (Marsh and Hurford, 1980).

Thus, knowing the magnetic field and electron temperature, the density of X-ray kernels is estimated from static equilibrium condition:

$$
\frac{B^{2}}{8 \pi}=N k T
$$

Substituting the values of $B, T$ and $k$ we obtain $N \simeq$ $5 \times 10^{12} \mathrm{~cm}^{-3}$. From the center frequency and bandwidth of the fine structure we estimate the magnetic field variation and also thickness for the kernel as given by

$$
\frac{\Delta B}{B} \simeq \frac{\Delta h}{L_{B}} \simeq \frac{\Delta f}{f}
$$

where $\Delta B$ is the magnetic field variation, $\Delta h$ is thickness of the kernel, $L_{B}=\left(\frac{1}{B} \frac{d B}{d h}\right)^{-1}$ is the magnetic scale height, $\Delta f$ is bandwidth of fine structure, and $f$ is the center frequency. The estimated variation of magnetic field through kernel is $\sim 150 \mathrm{G}$ for $\frac{\Delta f}{f} \sim 0.1$ and thickness of the kernel is $\sim 1.5 \times 10^{8} \mathrm{~cm}$ for the magnetic scale height, $L_{B}=10^{9} \mathrm{~cm}$.

We have calculated the optical depth to determine the range of angles at which the radiation is optically thin at $21 \mathrm{GHz}$ using Eq.9 in Zheleznyakov and Zlotnik (1988) paper:

$$
\tau_{j s}=\frac{s^{2 s}}{2^{s} s !} \frac{\pi e^{2}}{m c} \beta_{T}^{2 s-2} L_{B} \frac{N}{f} \frac{F_{j s}(\alpha)}{\sin \alpha}
$$

where $\beta_{T}$ is $=\sqrt{\frac{k T}{m c^{2}}} e, m$ and $c$ are electron charge and mass, and light velocity, in C.G.S. units respectively. $N$ is density, $f$ is observing frequency, $k$ is Boltzmann's constant and $T$ temperature of the kernel, and $F_{j s}(\alpha)$ is given by

$$
\frac{F_{j s}(\alpha)=\sin ^{2 s-2} \alpha\left(\sin ^{2} \alpha+2 s \cos ^{2} \alpha \pm \sqrt{\sin ^{4} \alpha+4 s^{2} \cos ^{2} \alpha}\right)^{2}}{|\cos \alpha|\left(\sin ^{4} \alpha+4 s^{2} \cos ^{2} \alpha \pm \sin ^{2} \alpha \sqrt{\sin ^{4} \alpha+4 s^{2} \cos ^{2} \alpha}\right)}
$$

where $\alpha$ is the angle between the line of sight and the magnetic field. Subscripts $j$ and $s$ are mode and harmonic, respectively.

Fig. 3 shows the variation of optical depth with the angle between the line of sight and the magnetic field for the 6th harmonic of the gyrofrequency at $21 \mathrm{GHz}$, $T=8 \times 10^{7} \mathrm{~K}, L_{B}=10^{9} \mathrm{~cm}$, and $N=10^{12}-10^{13} \mathrm{~cm}^{-3}$ in the kernel for the ordinary and extraordinary modes. According to the Fig. 3, the extraordinary mode - represented by the solid line - dominates the emission for both density values. For angle values greater than 2530 degrees, the ordinary mode is the dominant one. For the assumed values of the physical parameters, the optical depth is $<1$ for e-mode and o-mode if angle o? were to lie between about $10-15$ and 25 - 30 degrees, respectively, and beta plasma $\left(\frac{B^{2}}{8 \cdot \pi} / N . k . T<1\right)$. Hence the radiation can easily escape.

Some remarks has to be done. First, our 4 arcmin spatial resolution does not permit to determine the site of the hot kernel emission within the source. Second, 
frequency band coverage was narrow enough $(6 \mathrm{GHz}$ compared to the broad continuum from 1 up to 100 $\mathrm{GHz}$ emitted by the stronger bursts) to permit detection of just one line corresponding to a unique of the set of harmonics emitted by the source. Third, high temperature as $8 \times 10^{7} \mathrm{~K}$ gives a width of gyroresonance layer, that is of the order of the radiating source size. In this case, width of the layer is equal to source size over height. For the parameters we have taken the width of cyclotron line will be $50 \%$ wider than observed.

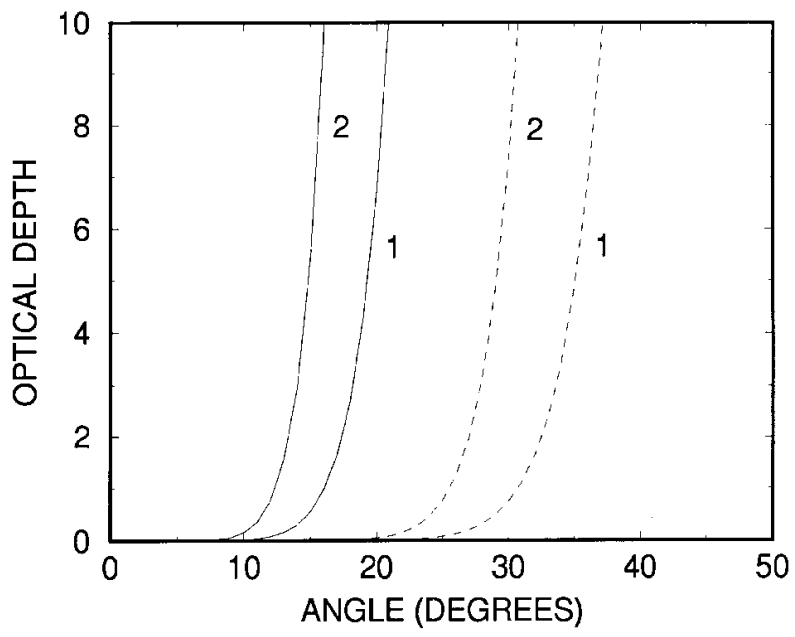

Figure 3. Optical depth variations at $21 \mathrm{GHz}$ with the angle between line of sight and magnetic field direction in the kernel are shown for densities $10^{12} \mathrm{~cm}^{-3}$, and $10^{13} \mathrm{~cm}^{-3}$ indicated by 1 and 2 for o-mode (dashed lines) and e-mode (continuous lines).

\section{Conclusions}

For the first time, observations are reported of narrow band $(2.0 \mathrm{GHz})$, short duration $(\leq 4 s)$, fine structure superposed on broad band burst observed in the frequency range of $18-23 \mathrm{GHz}$. This emission is suggested as due to the thermal radiation from hot kernel at the 6 th harmonic.

Assuming an upper limit of the kernel size as 4 arc seconds, the temperature of kernel is derived as about $8 \times 10^{7} \mathrm{~K}$. Also, we estimated a magnetic field of $\sim$ $1250 \mathrm{G}$, and electron density of $\sim 5 \times 10^{12} \mathrm{~cm}^{-3}$. From the centre frequency and bandwidth of the fine structure, the variation of magnetic field and thickness of the kernel were estimated as $150 \mathrm{G}$ and $1.5 \times 10^{8} \mathrm{~cm}$, respectively.

High spatial-time and frequency resolution observations over a wide band in microwaves with polarization are required to confirm our model.

\section{Acknowledgements}

We thank Dr. V.V. Zheleznyakov for useful discussions and comments about this work. Also, we acknowledge Drs. F.C.R. Fernandes and R.R. Rosa for useful discussions and suggestions. We thank the technical staff of Itapetinga Radio Observatory (ROI) and in particular R. Y. Hadano and E. Koppe Jr. for technical support on maintenance and functioning of the instrumentation. We acknowledge CNPq funding agency for financial support of K. R. Subramanian for the grant 300558/97-0 and also V. V. Zheleznyakov for the grant 452827/97-3 during this work. Finally, we thank Dr. R. P. Kane by reading and commenting the paper.

\section{References}

[1] V. Bogod, V. Garaimov, V.V. Zheleznyakov, E. Ya. Zlotnik, In: S. Pohjolainen (ed.) CESRA-Workshop on Coronal Explosive Events, p.71 (1998).

[2] J.R. Cecatto, In: Fine structure superimposed on mmwavelength $(18-23 \mathrm{GHz})$ spectra of solar active regions - Ms. Thesis, (INPE-5371 - TDI/470), São José dos Campos (1990).

[3] H.E. Coffey, In: H. E. Coffey (ed.) Solar Geophysical Data, 540-I, 112 (1989).

[4] C. De Jager, Solar Physics 64, 135 (1979).

[5] V.M. Fridman, B.N. Levin, O.A. Sheiner, Space Science Reviews 68, 253 (1994).

[6] O.G. Gontarev, A.P. Klassen, Solnechnie Dannye 1, 87 (1987).

[7] N. Gopalswamy, T.E.W. Payne, E.J. Schmahl, M.R. Kundu, J.R. Lemen, K.T. Strong, R.C. Canfield, J. de La Beaujardiere, Astrophysical Journal 437, 522 (1994).

[8] N.S. Kaverin, M.M. Kobrin, A.I. Korshunov, V.V. Shushunov, Solar Physics 63, 379 (1979).

[9] N.S. Kaverin, M.M. Kobrin, A.I. Korshunov, V.V. Shushunov, Soviet Astronomy 24(4), 442 (1980).

[10] A.P. Klassen, Solar Physics 138, 201 (1992).

[11] I.A. Krinberg, R.B. Teplitskaja, Solar Physics 25, 305 (1972).

[12] M.R. Kundu, K. Shibasaki, S. Enome, N. Nitta, Astrophysical Journal 431, L155 (1994).

[13] K.R. Lang, R.F. Willson, Advances in Space Research 2(11), 91 (1983)

[14] K.A. Marsh, G.J. Hurford, Astrophysical Journal 240 , L111 (1980).

[15] H.S. Sawant, J.R. Cecatto, B.R. Dennis, D.E. Gary, G.J. Hurford, Advances in Space Research 13(9), 191 (1993). 
[16] H.S. Sawant, J.R. Cecatto, Solar Physics 150, 375 (1994).

[17] H.S. Sawant, J.R. Cecatto, V.P. Nefedyev, A.M. Uralov, Solar Physics 152, 381 (1994).

[18] E. Schmahl, R.K. Shevgaonkar, M.R. Kundu, D. McConnell, Solar Physics 93, 305 (1984).

[19] R. Schwartz, Personal communication (1992).

[20] T. Shimizu, Publications of Astronomical Society of Japan 47, 251 (1995).

[21] M. Stahli, D.E. Gary, G.J. Hurford, Solar Physics 120, 351 (1989).
[22] M. Stahli, D.E. Gary, G.J. Hurford, Solar Physics 125, $343(1990)$.

[23] L. Van Driel-Gesztelyi, Solar Physics 163, 145 (1996).

[24] R.F. Willson, Astrophysical Journal 298, 911 (1985).

[25] V.V. Zheleznyakov, Yu.V. Tikhomirov, Solar Physics 81, 121 (1982).

[26] V.V. Zheleznyakov, E.Ya. Zlotnik, Soviet Astronomy Letters 14(3), 195 (1988). 